Rodrigo Peixoto Leite

Painel de automóveis populares: 0 design do cluster de direção sob o aspecto da ergonomia informacional

Dissertação apresentada como requisito parcial para obtenção do título de Mestre pelo Programa de PósGraduação em Design do Departamento de Artes \& Design da PUC-Rio.

Orientador: Cláudia Renata Mont’Alvão

Rio de Janeiro, abril de 2006. 


\title{
Painel de automóveis populares: 0 design do cluster de direção sob o aspecto da ergonomia informacional
}

\begin{abstract}
Dissertação apresentada como requisito parcial para obtenção do grau de Mestre pelo Programa de PósGraduação em Design do Departamento de Artes \& Design do Centro de Teologia e Ciências Humanas. Aprovada pela Comissão Examinadora abaixo assinada.
\end{abstract}

\author{
Profa. Dra. Cláudia Renata Mont’Alvão \\ Presidente/Orientadora - PUC-Rio
}

Prof. Dr. Vilmar Augusto Azevedo Miranda Membro - UFRJ

Profa. Dra. Luiza Helena Boueri Rebello Membro - Centro Universitário da Cidade

Prof. Dr. Paulo Fernando Carneiro de Andrade Coordenador Setorial do Centro de Teologia e Ciências Humanas - PUC-Rio 
Todos os direitos reservados. É proibida a reprodução total ou parcial do trabalho sem autorização da universidade, do autor e do orientador.

Rodrigo Peixoto Leite

Graduou-se em Desenho Industrial na PUC-Rio, na habilitação de Projeto de Produto (2002). Atua na área de design automotivo.

Ficha Catalográfica

Leite, Rodrigo Peixoto

Painel de automóveis populares : o design do cluster de direção sob o aspecto da ergonomia informacional / Rodrigo Peixoto Leite ; orientadora: Cláudia Renata Mont'Alvão . - Rio de Janeiro : PUC-Rio, Departamento de Artes e Design, 2006.

300 f. : il. (col.) ; $30 \mathrm{~cm}$

Dissertação (mestrado) - Pontifícia Universidade Católica do Rio de Janeiro, Departamento de Artes e Design.

Inclui referências bibliográficas.

1. Artes - Teses. 2. Ergonomia. 3. Painéis automotivos. 4. Design de automóveis. 5. Quadro de instrumentos. 6. Comandos automotivos. I. Mont'Alvão, Cláudia Renata. II. Pontifícia Universidade Católica do Rio de Janeiro. Departamento de Artes e Design. III. Título. 
Aos meus pais, Alberto e Dalva Leite, meus avós e a todos que em vida sempre me incentivaram a acreditar em meus sonhos. Dedico também ao grande arquiteto brasileiro, tio e amigo Antônio Rosas (in memorium). 


\section{Agradecimentos}

A Deus por me permitir iniciar e concluir mais esta etapa em minha vida.

A minha orientadora à Professora Cláudia Renata Mont'Alvão pela confiança e incentivo ao meu trabalho.

Aos meus pais, Alberto e Dalva e minha avó Jacyra pela força, carinho e incentivo para comigo.

Aos Professores Anamaria de Moraes, Cláudio de Freitas Magalhães, Rita Maria Couto, Neville Larica, Manuela Quaresma, Leônidas Moraes e Rejane Spitz pelo estímulo constante.

Ao meu tio Hélio Leite por ter cedido o espaço para a construção do simulador e seu computador para o término este trabalho.

Aos amigos Adriano Abreu, Pedro Halbritter, Eduardo Brandão, Christian Smith, Aylton Pessanha, e tantos outros que colaboraram em fases distintas da pesquisa.

Aos meus amigos de infância, sempre próximos, pelo carinho, paciência e incentivo ao meu trabalho.

Aos professores da comissão examinadora.

Aos voluntários que participaram da pesquisa.

Aos funcionários do Departamento de Artes \& Design da PUC-Rio.

Ao Conselho Nacional de Desenvolvimento Científico e Tecnológico pelo auxílio concedido para o desenvolvimento da pesquisa. 


\section{Resumo}

LEITE, Rodrigo Peixoto. MONT'ALVÃO, Claudia Renata (Orientador). Painel de automóveis populares: o design do cluster de direção sob o aspecto da ergonomia informacional. Rio de Janeiro, 2006. 300 p. Dissertação de Mestrado - Departamento de Artes, Pontifícia Universidade Católica do Rio de Janeiro.

A evolução do design de automóveis possui características interessantes, sob o ponto de vista da relação estético-funcional estabelecida em cada época. No início, os projetos de veículos únicos priorizavam a funcionalidade em detrimento do bem-estar do usuário. Com o tempo, esta maneira de se projetar foi sendo modificada, passando por períodos que valorizaram estritamente o desempenho, a aerodinâmica e a forma para um modelo onde a relação humano-máquina é o centro principal de estudo. Com novos e diferentes modelos de veículos, estes passaram a apresentar variações no posicionamento e projeto de comandos e mostradores, principalmente na região do cluster de direção. Desta forma, o presente estudo objetivou demonstrar que esta variação do posicionamento e de projeto gera confusão e erros de julgamento por parte do usuário, podendo ocasionar graves acidentes e incidentes durante a realização da tarefa, principalmente àquele que não está acostumado com o sistema a ser utilizado. Para comprovar esta hipótese, foi elaborado um estudo que, primeiramente, buscou entender o desenvolvimento dos comandos e mostradores automotivos e o uso de normas e recomendações de projeto para traçar um panorama da disposição das informações no interior dos veículos. Para o desenvolvimento da pesquisa, determinou-se o enfoque na categoria "popular". O estudo do surgimento desta categoria, do panorama atual e do perfil do consumidor, contribuiu para se tentar observar o modelo mental dos usuários em relação ao posicionamento dos instrumentos. Para isso foram aplicadas diferentes técnicas, tais como um estudo comparativo dos modelos; a aplicação de questionários com usuários motoristas; e a realização de um grupo focal. O levantamento de dados contemplou ainda a realização de testes em um simulador real de interior de veículo. Outrossim, foi possível observar constrangimentos causados pela variação do projeto e pelo posicionamento de um grupo de comandos/mostradores do cluster e sugerir recomendações para projetos futuros.

\section{Palavras-chave}

Ergonomia; painéis automotivos; design de automóveis; quadro de instrumentos, comandos automotivos. 


\section{Abstract}

LEITE, Rodrigo Peixoto; MONT'ALVÃO, Cláudia Renata (Advisor). Automobile panel: cluster's design according to informational ergonomics. Rio de Janeiro, 2006. 300 p. MSc. Dissertation Departamento de Artes \& Design, Pontifícia Universidade Católica do Rio de Janeiro.

The evolution of automobiles design has interesting characteristics, under the point of view of the aesthetic-functionary relation established at each time. In the beginning, the projects of unique vehicles prioritized the functionality in detriment of well-being of the user. As time passed by, this way of projecting were being modified, passing by periods that had strict valued the performance, the aerodynamics and the form for a model which the human being-machine relation is the main center of study. With new and different models of vehicles, these had started to present variations in the positioning and project of commands and counters, mainly in the region of cluster. In this way, the present study aimed to demonstrate that this variation of the positioning and project generates confusion and errors of judgment by the user, and can cause serious accidents and incidents during the accomplishment of the task, mainly to that user that is not familiar with the system to be used. To state this hypothesis, a study was elaborated that, first, searched to understand the automotive development of the commands and counters and the use of norms and recommendations of project to trace a panorama of the disposal of the information in the interior of the vehicles. For the development of the research, the approach in "the popular" category was determined. The study of the sprouting of this category, the current panorama and the profile of the consumer, it contributed to try itself to observe the mental model of the users in relation to the positioning of the instruments. For this different techniques had been applied, such as a comparative study of the models; the application of questionnaires with drivers; and the realization of a focus group. The data-collecting still contemplated the accomplishment of tests in a real simulator of vehicle interior. Thus, it was possible to observe constraints caused by the variation of the project and the positioning of a group of commands/displays in the cluster and to suggest recommendations for future projects.

\section{Keywords}

Ergonomics and automotive panels; automobile design; instrument cluster; automotives commands. 


\section{Sumário}

11 Introdução

2 A Questão da segurança segundo a ergonomia e os painéis de veículos

2.1. O desenvolvimento dos painéis de instrumentos na $2^{\underline{a}}$ Guerra Mundial

2.2. A ergonomia aplicada aos painéis automotivos: analisando o automóvel como um produto 26

2.2.1. A Convergência de tecnologias 29

2.2.2. Usabilidade nos painéis de automóveis 31

2.2.3. Normas e recomendações no desenvolvimento de painéis automotivos 34

2.3. Variações de comandos e mostradores de painéis automotivos 38

2.4. Conclusão do capítulo 50

3 O carro popular no Brasil $\quad 51$

3.1. O automóvel no Brasil $\quad 51$

3.2. Antecedentes do carro popular no Brasil e no mundo 70

3.2.1. O DKW Vemag

3.2.2. Willys Dauphine/Gordine 75

3.2.3. O Fusquinha 77

3.2.4. Ford Corcel 79

3.2.5. Fiat 147

3.3. O conceito de carro popular no Brasil 82

3.4. O mercado atual brasileiro de veículos populares 88

3.4.1. Volkswagen (Gol/Parati/Fox) 89

$\begin{array}{ll}\text { 3.4.2. Ford (Ka/Fiesta) } & 91\end{array}$

3.4.3. Chevrolet (Corsa/Celta) 93

3.4.4. Fiat (Uno/Palio/Siena) 94

3.4.5. Peugeot (206) 95

3.4.6. Renault (Clio/Kangoo) 96

3.4.7. Hyundai (Atos) 96

3.5. O perfil do consumidor dos "populares" 97

3.6. A interferência do consumidor nos carros populares: customização, personalização ou "tuning". 
4 Delineamento da pesquisa 109

4.1. Tema 109

4.2. Problema 110

4.3. Hipótese 110

4.4. Variáveis 111

4.4.1. Variáveis independentes 111

4.4.2. Variáveis dependentes 112

4.5. Objeto da pesquisa 112

4.6. Objetivo Geral 112

4.7. Justificativa 112

4.8. Recorte da pesquisa 113

5 Método, técnicas e procedimentos da pesquisa 116

5.1. Estudo comparativo 117

5.1.1. Peugeot 206 Sensation $1.02 \mathrm{p}$. 118

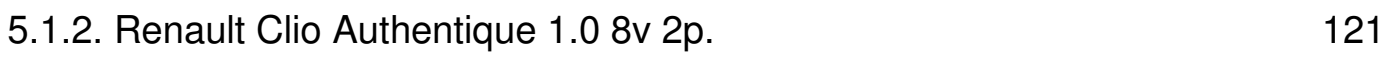

5.1.3. Ford Novo Fiesta 1.0I Personnalite 124

5.1.4. Fiat Novo Palio EX 1.0 8v. 2p. 127

5.1.5. Chevrolet Novo Corsa Hatch VHC Joy 1.0 4p. 129

5.1.6. Volkswagen Fox City 1.0

5.2. Caixa morfológica 136

5.3. Test-Drive 146

5.3.1. Test-drive Peugeot 206 Sensation $1.02 p$. 150

5.3.2. Test-drive Renault Clio Authentique 1.0 8v 2p. 152

5.3.3. Test-drive Novo Palio EX $1.08 \mathrm{v}$ 2p. 154

5.3.4. Test-drive Novo Fiesta 1.0I Personnalite 155

5.3.5. Test-drive Fox City $1.0 \quad 158$

5.3.6. Test-drive Novo Corsa Hatch VHC Joy $1.04 p$.

5.4. Questionários 161

5.4.1. Aplicação do questionário 163

5.4.2. Vantagens do uso de questionário 163

5.4.3. Desvantagens do uso de questionário 163

5.4.4. Aplicação do questionário estruturado 164

5.5. Grupo de foco 166

5.5.1. Vantagens e desvantagens do Grupo de Foco 167 
5.5.2. Utilização do Grupo de Foco

5.5.3. Planejamento do Grupo de Foco 170

5.5.4. Tratamento dos dados 175

5.5.5. Aplicação do Grupo de Foco 175

5.6. Observação simulada através do uso de simulador 177

5.6.1. O Simulador F741 179

5.6.2. Construção do Simulador F741 179

5.6.3. Seleção da amostra 189

5.6.4. Aplicação do questionário semi-estruturado 189

5.6.5. Descrição do experimento 190

6 Resultados e análise dos dados obtidos 194

6.1. Estudo comparativo 194

$\begin{array}{ll}\text { 6.2. Test-drive } & 197\end{array}$

6.3. Questionário estruturado 198

6.4. Grupo de foco 200

6.5. Questionário semi-estruturado 207

6.6. Tarefa simulada 217

7 Recomendações e discussão final 225

7.1. Introdução 225

7.1.1. Velocímetro 226

7.1.2. Tacômetro 227

7.1.3. Velocímetro e tacômetro acoplados 227

7.1.4. Inversão de posicionamento entre tacômetro e velocímetro 228

7.1.5. Mostrador do nível de combustível no tanque 228

7.1.6. Mostrador do nível de temperatura 229

7.1.7. Inversão de posicionamento entre marcador do nível de combustível e do nível de temperatura 229

7.1.8. Luzes-espia 230

7.1.9. Observações em geral 230

7.2. Recomendações propostas 231

7.3. Lições aprendidas e considerações finais 234

7.4. Desdobramentos da pesquisa 236

8 Referências bibliográficas $\quad 237$ 
10 Apêndices

247

10.1. Tabela de comparativo de equipamentos dos veículos selecionados

10.2. Questionário estruturado

10.3. Resultados do questionário estruturado

10.4. Roteiro para perguntas do grupo de foco 260

10.5. Transcrição do grupo de foco

261

10.6. Questionário semi-estruturado

287

10.7. Resultados do questionário semi-estruturado

290

10.8. Planta da sala onde foi realizado o teste simulado

292

10.9. Arranjos de clusters produzidos na tarefa simulada

293

10.10. Resultados da tarefa simulada 


\section{Lista de figuras}

Figura 1 - Painel do avião DC-7 (RUUD LEEUW, [2006]). 22

Figura 2 - Painel do avião Superconstellation (WIN JACK'S [2005]). 23

Figura 3 - Cabine do maquinista (HESKETT, 1998), p. 167.

Figura 4 - Fiat Stilo Connect: convergência de sistemas tecnológicos. 30

Figura 5 - Comandos do tipo thumb wheel. 40

Figura 6 - Exemplo de comando do tipo alavanca, à esquerda, painel do Monza

(MANUAL DO PROPRIETÁRIO DO MONZA, 1984) e à direita, do Uno. 40

Figura 7 - Comandos de deslizamento lateral. $\quad 41$

Figura 8 - Comandos do tipo botão giratório. $\quad 41$

Figura 9 - Comandos do tipo botão de pressão. 42

Figura 10 - Comandos do tipo interruptor: Lada Laika e Fiat 147.

Figura 11 - Alavancas: controles de liga/desliga ou gradação (SAE J1139). 45

Figura 12 - Comandos dos vidros elétricos: Gol GTi 1989, à esquerda e Parati

GLS 1992.

Figura 13 - Comandos dos vidros elétricos: Gol GTi 1994, à esquerda (QUATRO

RODAS, 1994) e Prêmio (QUATRO RODAS, 1998). 46

Figura 14 - Cluster com odômetro digital do Kadett GSi (MANUAL DO

PROPRIETÁRIO DO KADETT/IPANEMA, 1992). 49

Figura 15 - Peugeot Type 3 (PEUGEOT FANS CLUB, [2004]). 52

Figura 16 - 1a fábrica da Ford no país, em 1919 (QUATRO RODAS, 2000). 54

Figura 17 - Inauguração da fábrica da GM no Brasil (AUTOMÓVEL \&

REQUINTE, 2001). 56

Figura 18 - Apresentação do Ford Galaxie em São Paulo (QUATRO RODAS, 1990b). 59

Figura 19 - VW 1600 "Zé do Caixão" e TL. $\quad 60$

Figura 20 - Um dos primeiros Chevette lançado no país. 62

Figura 21 - Painel do Chevette 1973. Adaptado (QUATRO RODAS, 1973). 62

Figura 22 - Cluster do Chevette. 63

Figura 23 - Visão do interior da Brasília. $\quad 64$

Figura 24 - Teste do Fiat 147 na Ponte Rio-Niterói (QUATRO RODAS, 1978). 65

Figura 25 - Um dos primeiros modelos do VW Gol. 66

Figura 26 - Painel do Gol década de 80.66

Figura 27 - Chevrolet Monza e Opel Ascona (BILLING AUTOS, [2005]). 68 
Figura 28 - Jaguar MK 1959 com freios ABS (HERITAGE CLASSICS, [2004]). 68

Figura 29 - Demonstração da Cadillac (HESKETT, 1998), p.71. 71

Figura 30 - Painel de instrumentos da Vemaguet (QUATRO RODAS, 1978). 73

Figura 31 - Comandos da Vemaguet (QUATRO RODAS, 1962), p.92. 73

Figura 32 - DKW Vemaguet Pracinha. $\quad 74$

Figura 33 - Gordini e seu painel. 76

Figura 34 - Fusca "Pé-de-Boi" (BEST CARS, 2001). 78

Figura 35 - Corcel I e seu painel. 79

Figura 36 - Painel do Fiat 147.

Figura 37 - Cluster do Fiat 1471977.

Figura 38 - Anúncio do Ford LTD 1968. (AMIGOS DO GALAXIE, [2004]). 83

Figura 39 - Renault R8. (R8 CLUB, 2005) e Fiat Uno Mille (DIVULGAÇÃO). 84

Figura 40 - Satélites do painel do Uno Mille. 85

Figura 41 - Gol 1000: vidro colado (QUATRO RODAS, 1992) e cluster. $\quad 86$

Figura 42 - Cluster do Fusca "Itamar".

Figura 43 - Gol Special e City: seria a aplicação do Styling? (DIVULGAÇÃO). 90

Figura 44 - Ford Ka e seu cluster. 92

Figura 45 - Chevrolet Celta e o detalhe da tampa de combustível. 94

Figura 46 - Fiat Uno Mille Fire e seu cluster. $\quad 94$

Figura 47 - Hyundai Atos Prime. $\quad 97$

Figura 48 - Fusca-57 e Fusca-Rolls-Royce. 102

Figura 49 - Moncedes 190E e Monza Conversível. 102

Figura 50 - "Tunados": Fusca com faróis do Ford Ka e Chevette Junior. 103

Figura 51 - Tuning popular no Fiat 147 e o de fábrica, no Gol GTI. 104

Figura 52 - Gol 1000 original e a versão "tunada". 104

Figura 53 - Belina caracterizada como off-road e a Belina original. 105

Figura 54 - Gol Trend e Cross Fox. 105

Figura 55 - Personalização do cluster. 106

Figura 56 - Cluster do Peugeot $206 . \quad 119$

Figura 57 - Cluster aceso do Peugeot $206 . \quad 119$

Figura 58 - Panorama do painel do Peugeot 206.

Figura 59 - Display central MTN e botão do pisca-alerta. 121

Figura 60 - Cluster do Renault Clio. 123

Figura 61 - Panorama do painel do Renault Clio. 123

Figura 62 - Cluster do Ford Fiesta. 125

Figura 63 - Panorama do painel do Fiesta brasileiro, à esquerda, e europeu 
(REPORT MOTORI, 2004).

Figura 64 - Cluster do Fiat Novo Palio. 128

Figura 65 - Panorama do painel do Novo Palio. 129

Figura 66 - Panorama do Painel do Novo Corsa. 130

Figura 67 - Cluster do Novo Corsa. 131

Figura 68 - Comando de acionamento de farol do Novo Corsa. 132

Figura 69 - Fox City. 133

Figura 70 - Painel do Corsa 1994, à esquerda, e do Fox City. 134

Figura 71 - Cluster do Fox City. 135

Figura 72 - Propaganda do Fiesta Class. 156

Figura 73 - Detalhe da linha de cintura do Novo Fiesta. 157

Figura 74 - Desenho do painel do Fox. 159

Figura 75 - Mudança de nomenclatura dos veículos no questionário piloto. 165

Figura 76 - Divulgação do questionário no site de relacionamentos Orkut. 165

Figura 77 - Alguns rascunhos conceituais para a construção do simulador. 180

Figura 78 - Fiat 147 GL 1978 utilizado na construção do simulador. 180

Figura 79 - Desmontagem do veículo. 181

Figura 80 - Retirada de peças. 182

Figura 81 - Corte do veículo. 182

Figura 82 - Separação das peças cortadas. 183

Figura 83 - Retirada de parte do chassi. 183

Figura 84 - Preparação do chassi. 184

Figura 85 - Acabamentos em geral. 184

Figura 86 - Imagem da ante-sala. $\quad 185$

Figura 87 - Acoplagem das peças fixas. 185

Figura 88 - Construção do painel. 187

Figura 89 - Painel do simulador. $\quad 187$

Figura 90 - Panorama do simulador com as peças móveis. 188

Figura 91 - Visão superior do Simulador F741. 188

Figura 92 - Ante-sala: participantes aguardando o momento do teste. $\quad 190$

Figura 93 - Participantes preenchendo o questionário semi-estruturado. 191

Figura 94 - Participantes observando os mostradores disponíveis. 191

Figura 95 - Participantes selecionando os mostradores. 192

Figura 96 - Participantes montando o cluster de direção. 192

Figura 97 - Exemplo de configuração de cluster escolhida pelos usuários. 193

Figura 98 - Distribuição por veículos. 198 
Figura 99 - Motorização dos veículos dos participantes.

Figura 100 - Tempo de uso do veículo. 208

Figura 101 - Tempo de uso diário do veículo. 208

Figura 102 - Observação do tacômetro. 209

Figura 103 - Observação do velocímetro. 210

Figura 104 - Percentual de homens e mulheres respondentes. 210

Figura 105 - Faixa etária dos participantes. 211

Figura 106 - Grau de instrução dos respondentes. 211

Figura 107 - Tempo de habilitação dos respondentes. 212

Figura 108 - Exemplo de pontos positivos destacado pelo respondente. 213

Figura 109 - Exemplo de pontos positivos destacado pelo respondente. 213

Figura 110 - Exemplo de pontos positivos destacado pelo respondente. 213

Figura 111 - Destaque dos pontos negativos de um respondente. 214

Figura 112 - Destaque dos pontos negativos de um respondente. 214

Figura 113 - Destaque dos pontos negativos de um respondente. 215

Figura 114 - Destaque dos pontos negativos de um respondente. 215

Figura 115 - Posicionamento do símbolo de combustível Peugeot 206.

Figura 116 - Composição com os instrumentos mais utilizados. 217

Figura 117 - Questionário estruturado via internet. 252

Figura 118 - Questionário estruturado via internet. 252

Figura 119 - Questionário estruturado via internet. 253

Figura 120 - Questionário estruturado via internet. 253

Figura 121 - Faixa etária. 254

Figura 122 - Percentual de homens e mulheres respondentes. 254

Figura 123 - Distribuição por Estados. 254

Figura 124 - Distribuição por veículo. 254

Figura 125 - Fox: Capacidade de localização dos comandos do painel. 254

Figura 126 - 206: Capacidade de localização dos os comandos do painel. 254

Figura 127 - Clio: Capacidade de localização dos comandos do painel. 255

Figura 128 - Corsa: Capacidade de localização dos comandos do painel. 255

Figura 129 - Palio: Capacidade de localização dos comandos do painel. 255

Figura 130 - Fiesta: Capacidade de localização dos comandos do painel. 255

Figura 131 - Fox: Conhecimento das funções dos comandos do painel. 255

Figura 132 - 206: Conhecimento das funções dos comandos do painel. 255

Figura 133 - Clio: Conhecimento das funções dos comandos do painel. 256

Figura 134 - Corsa: Conhecimento das funções dos comandos do painel. 256 
Figura 135 - Palio: Conhecimento das funções dos comandos do painel. 256

Figura 136 - Fiesta: Conhecimento das funções dos comandos do painel. 256

Figura 137 - Pré-disposição dos usuários para participar de uma entrevista. 256

Figura 138 - Faixa etária. 257

Figura 139 - Percentual de homens e mulheres repondentes. 257

Figura 140 - Pré-disposição dos usuários para participar de uma entrevista. 257

Figura 141 - Distribuição por veículo. 257

Figura 142 - Fox: Capacidade de localização dos comandos do painel. $\quad 257$

Figura 143 - 206: Capacidade de localização dos comandos do painel. 258

Figura 144 - Clio: Capacidade de localização dos comandos do painel. 258

Figura 145 - Corsa: Capacidade de localização dos comandos do painel. 258

Figura 146 - Palio: Capacidade de localização dos comandos do painel. 258

Figura 147 - Fiesta: Capacidade de localização dos comandos do painel. 258

Figura 148 - Fox: Conhecimento das funções dos comandos do painel. 258

Figura 149 - 206: Conhecimento das funções dos comandos do painel. 259

Figura 150 - Clio: Conhecimento das funções dos comandos do painel. 259

Figura 151 - Corsa: Conhecimento das funções dos comandos do painel. 259

Figura 152 - Palio: Conhecimento das funções dos comandos do painel. 259

Figura 153 - Fiesta: Conhecimento das funções dos comandos do painel. 259

Figura 154 - Veículo Próprio. 290

Figura 155 - Tipo de motorização. 290

Figura 156 - Tempo de uso do veículo. 290

Figura 157 - Horas ao dia dirigindo. 290

Figura 158 - Identificação dos comandos mostradores do painel. 290

Figura 159 - Dificuldade na identificação dos comandos e mostradores. 290

Figura 160 - Equívocos de leitura. 291

Figura 161 - Observação do conta-giros. 291

Figura 162 - Observação do velocímetro. 291

Figura 163 - Percentual de homens e mulheres repondentes. 291

Figura 164 - Faixa etária. $\quad 291$

Figura 165 - Grau de escolaridade. 291

Figura 166 - Tempo de habilitação. 291

Figura 167 - Freqüência de uso de cada mostrador do velocímetro. 295

Figura 168 - Freqüência de uso do velocímetro quanto ao tipo deste. 295

Figura 169 - Freqüência de uso do tacômetro. 295

Figura 170 - Freqüência de uso cada tacômetro. 296 
Figura 171 - Freqüência de uso do tacômetro por tipo de escala. 296

Figura 172 - Freqüência de uso mostrador de temperatura. 296

Figura 173 - Freqüência de uso de cada mostrador do nível de temperatura. 296 Figura 174 - Freqüência de uso de cada mostrador do nível de combustível. 297 Figura 175 - Freqüência de uso do mostrador do nível de combustível por tipo de escala.

Figura 176 - Posicionamento velocímetro versus tacômetro.

Figura 177 - Freqüência do posicionamento do marcador de combustível (lateralidade).

Figura 178 - Freqüência do posicionamento do marcador de combustível (verticalidade).

Figura 179 - Freqüência do posicionamento do marcador de combustível (lateralidade).

Figura 180 - Freqüência do posicionamento do marcador de combustível (verticalidade).

Figura 181 - Freqüência do posicionamento do marcador de combustível em relação ao tacômetro e velocímetro.

Figura 182 - Freqüência do posicionamento do marcador de temperatura em relação ao tacômetro e velocímetro.

Figura 183 - Orientação do marcador de combustível. 300

Figura 184 - Orientação do marcador de temperatura. 


\section{Lista de tabelas}

Tabela 1 - Produtos tradicionais versus modernos. JURAN \& GRYNA (1992 apud FIOD NETO et al., 1998).

Tabela 2 - Controles de liga/desliga ou gradação (SAE J1139).

Tabela 3 - Controles do tipo interruptor para vidros elétricos (SAE J1139).

Tabela 4 - Empresas pioneiras. In (ORSINI,1967 e SHAPIRO, 1994 apud FONSECA, 1996).

Tabela 5 - Exemplo da técnica da Caixa Morfológica (BOMFIM, 1995). 138

Tabela 6 - Características dos velocímetros. 139

Tabela 7 - Características dos tacômetros. 141

Tabela 8 - Características dos marcadores do nível de combustível. 142

Tabela 9 - Características dos marcadores do nível de temperatura do líquido de arrefecimento do motor.

Tabela 10 - Informações sobre os participantes do Grupo de Foco. 176

Tabela 11 - Velocímetros dos veículos selecionados. 218

Tabela 12 - Tacômetros dos veículos selecionados. 218

Tabela 13 - Mostradores de temperatura dos veículos selecionados. 219

Tabela 14 - Mostradores de combustível dos veículos selecionados. 220 